\title{
Correction to: A feasibility analysis of the ArcBlate MR-guided high-intensity focused ultrasound system for the ablation of uterine fibroids
}

\author{
Chin-Jung Wang ${ }^{1,2} \cdot$ Gigin Lin $^{2,3} \cdot$ Yi-Ting Huang ${ }^{1} \cdot$ Cindy Hsuan Weng ${ }^{1} \cdot$ Kai-Yun Wu ${ }^{1,2} \cdot$ Yu-Ying Su ${ }^{1}$ Yu-Shan Lin ${ }^{1}$. \\ Kit-Sum Mak ${ }^{1}$
}

Published online: 22 September 2021

(c) Springer Science+Business Media, LLC, part of Springer Nature 2021

\section{Correction to: Abdominal Radiology https://doi.org/10.1007/s00261-021-03203-8}

The original version of this article has an error in the reference citation. The Reference 11-14 are indeed not suitable and have been corrected. In addition, Reference 26 and 33; Reference 29 and 32 are the same so it is also corrected with this correction. The corrected details are given below.

(a) Page 1: In the 10th line, the second paragraph of under the heading "Introduction" should be changed from "Noninvasive high-intensity focused ultrasound (HIFU) ablation has emerged as an alternative option to surgery for uterine fibroids, offering minimal invasiveness without an incision that can be performed as a uterineconserving outpatient treatment in most types of uterine fibroids [11-14]." to "Noninvasive high-intensity focused ultrasound (HIFU) ablation has emerged as an alternative option to surgery for uterine fibroids, offering minimal invasiveness without an incision that can be performed as a uterine-conserving outpatient treatment in most types of uterine fibroids [11-13]."

(b) Page 2: In the 2nd line of Introduction section should be changed from "Compared to the uterine artery embo-

The original article can be found online at https://doi.org/10.1007/ s00261-021-03203-8.

Chin-Jung Wang

wang2260@gmail.com

1 Division of Gynecologic Endoscopy, Department of Obstetrics and Gynecology, Chang Gung Memorial Hospital at Linkou, Taoyuan, Taiwan

2 Chang Gung University College of Medicine, Taoyuan, Taiwan

3 Department of Medical Imaging and Intervention, Chang Gung Memorial Hospital at Linkou, Taoyuan, Taiwan lization, another minimalinvasive treatment option, HIFU is preferred if clinicians and patients have concerns for low vascularity fibroids, high risk for anesthesia/sedation, radiation exposure, impaired renal function, allergy to iodine contrast medium, bleeding tendency, or difficult vascular access [15-17]." to "Compared to the uterine artery embolization, another minimalinvasive treatment option, HIFU is preferred if clinicians and patients have concerns for low vascularity fibroids, high risk for anesthesia/sedation, radiation exposure, impaired renal function, allergy to iodine contrast medium, bleeding tendency, or difficult vascular access $[12,14,15] . "$

(c) Page 2: In the 5th line under the heading "Introduction" should be changed from "Localized ablation is achieved by concentrating acoustic waves at a focal point to cause heat and raise focal temperature to over $55^{\circ} \mathrm{C}$, without affecting the surrounding tissues [18, 19]." to "Localized ablation is achieved by concentrating acoustic waves at a focal point to cause heat and raise focal temperature to over $55^{\circ} \mathrm{C}$, without affecting the surrounding tissues $[16,17] . "$

(d) Page 3: In the second paragraph under the heading "Patients" heading should be changed from "The diagnostic criteria for adenomyosis using MRI included (1) low signal intensity of myometrium, with indistinct margins and (2) diffuse or focal junctional zone exceeding $12 \mathrm{~mm}$ [20]." to "The diagnostic criteria for adenomyosis using MRI included (1) low signal intensity of myometrium, with indistinct margins and (2) diffuse or focal junctional zone exceeding $12 \mathrm{~mm}$ [18]."

(e) Page 3: Under the heading "Pretreatment screening and preparation" should be changed from "Other preprocedural preparations included an 8 -h fasting period and enema administrations to exclude air and other possible obstacles (e.g., bowel loops anterior to the 
uterus), and abdominal and pubic hair shaving to allow the skin to connect with the ultrasound beam closely to prevent the skin burn [21-23]." to "Other pre-procedural preparations included an 8-h fasting period and enema administrations to exclude air and other possible obstacles (e.g., bowel loops anterior to the uterus), and abdominal and pubic hair shaving to allow the skin to connect with the ultrasound beam closely to prevent the skin burn [19-21],"

(f) Page 4: Under the heading "MRgHIFU treatment" should be changes from "These temperature maps were calculated from raw data centered in the k-space matrix and based on the proton resonance frequency shift [24, 25]," to "These temperaturemaps were calculated from raw data centered in the k-space matrix and based on the proton resonance frequency shift [22, 23],"

(g) Page 4: Under the heading "Assessments and data analysis"

i) "Classification of the benign uterine tumor by fibroid (i.e., subserosal, intramural, submucosal, or adenomyosis) and Funaki type was based on the signal intensity of the T2-weighted MR images [26]," should read as "Classification of the benign uterine tumor by fibroid (i.e., subserosal, intramural, submucosal, or adenomyosis) and Funaki type was based on the signal intensity of the T2-weighted MR images [24],"

ii) "The SF-36 questionnaire was used for evaluating quality of life as described in the previous research. Higher scores indicated better quality of life [27]." should read as "The SF-36 questionnaire was used for evaluating quality of life as described in the previous research. Higher scores indicated better quality of life [25]."

\section{(h) Page 5, 6 and 7: Under heading "Discussion"}

(i) "Despite the non-invasive feature of HIFU, many limitations related to technique and mechanical design have prevented its widespread application [22, 28]." should read as "Despite the non-invasive feature of HIFU, many limitations related to technique and mechanical design have prevented its widespread application [20, 26]."

(ii) "Current MRgHIFU treatment protocols suggest an ablation rate (i.e., nonperfused volume [NPV] ratio) of $70-80 \%$ to achieve the desired treatment outcomes [21, 29]." should read as "Current MRgHIFU treatment protocols suggest an ablation rate (i.e., nonperfused volume [NPV] ratio) of 70-80\% to achieve the desired treatment outcomes [19,27]."

(iii) "The effectiveness of ArcBlate MRgHIFU seemed not to be inferior to that of other MRgHIFU systems, which demonstrated mean fibroid volume shrinkage rates of $12.6-18.1 \%$ at 3 months [30, 31]." should read as "The effectiveness of ArcBlate MRgHIFU seemed not to be inferior to that of other MRgHIFU systems, which demonstrated mean fibroid volume shrinkage rates of $12.6-18.1 \%$ at 3 months [28, 29]."

(iv) "A higher NPV ratio was found to be correlated with greater tumor volume shrinkage. Keserci et al. discovered that if the NPV achieved a ratio of at least $90 \%$, the mean fibroid reduction ratio would be as high as $54 \pm 13 \%$ [31]. A report of 80 patients with leiomyoma suggested that a larger NPV ratio would result in greater shrinkage and improved relief of symptoms [32]." should read as "A higher NPV ratio was found to be correlated with greater tumor volume shrinkage. Keserci et al. discovered that if the NPV achieved a ratio of at least $90 \%$, the mean fibroid reduction ratio would be as high as $54 \pm 13 \%$ [29]. A report of 80 patients with leiomyoma suggested that a larger NPV ratio would result in greater shrinkage and improved relief of symptoms [27]."

(v) "According to the literature, treating Funaki type 3 fibroid tissue by HIFU is difficult because of highvascularization which results in decreasing therapeutic effects of heating [23,33,34], yet our intramural case was identified to be Funaki type 3." should read as "According to the literature, treating Funaki type 3 fibroid tissue by HIFU is difficult because of high vascularization which results in decreasing therapeutic effects of heating [21, 24, 30], yet our intramural case was identified to be Funaki type 3."

(vi) "Despite the decreased estrogen level in this patient (49 years) who approached menopause may also have influenced the treatment outcome [35]," should read as "Despite the decreased estrogen level in this patient (49 years) who approached menopause may also have influenced the treatment outcome [31]," (vii) "No real-time monitoring in thermal damage in tissue was one of the limitations in ultrasonographyguided HIFU therapy [36], while it could be resolved under MR guidance. Besides, patients having pelvic endometriosis, adhesions locating between the uterus and bowel, or $>10-\mathrm{mm}$ abdominal surgical scar are not recommended to receive HIFU therapy [37]. However, one patient with adenomyosis in our study showed a lower shrinkage rate after 3 months than another study (our study vs. another study: $5.5 \%$ vs. $46.3 \%$ ) [38]." should read as "No real-time monitoring in thermal damage in tissue was one of the limitations in ultrasonography-guided HIFU therapy [32], while it could be resolved under MR guidance. Besides, patients having pelvic endometriosis, adhesions locating between the uterus and bowel, or $>10-\mathrm{mm}$ abdominal surgical 
scar are not recommended to receive HIFU therapy [33]. However, one patient with adenomyosis in our study showed a lower shrinkage rate after 3 months than another study (our study vs. another study: 5.5\% vs. $46.3 \%$ ) [34]."

(viii) "[39] Considering the treatment outcome among patients by Funaki classification, quality of life scores in the type 3 cohort seemed to decrease slightly after the treatment," should read as "Considering the treatment outcome among patients by Funaki classification, quality of life scores in the type 3 cohort seemed to decrease slightly after the treatment,"

(ix) "The most common adverse effect of HIFU treatment is skin burn [30, 39];" should read as "The most common adverse effect of HIFU treatment is skin burn [28, 35];"’

(x) "Much research has shown that rectal and bladder filling are very useful practices for avoiding adverse effects and for mitigating bowelrelated side effects after MRgHIFU treatment [40, 41]." should read as "Much research has shown that rectal and bladder filling are very useful practices for avoiding adverse effects and for mitigating bowelrelated side effects after MRgHIFU treatment [11,36]."

(xi) "In addition, endometrial ablation has been used to treat submucosal fibroids [42] but it may cause severe endometrial impairment and reduce possibilities of future pregnancy [43]." should read as "In addition, endometrial ablation has been used to treat submucosal fibroids [37] but it may cause severe endometrial impairment and reduce possibilities of future pregnancy [38]."

\section{References}

1. Prayson RA, Hart WR (1995) Pathologic considerations of uterine smooth muscle tumors. Obstet Gynecol Clin North Am. 22 (4):637-657.

2. Stewart EA (2001) Uterine fibroids. Lancet. 357 (9252):293-298.

3. Wise LA, Laughlin-Tommaso SK (2016) Epidemiology of uterine fibroids: from menarche to menopause. Clin Obstet Gynecol. 59 (1):2-24

4. Okolo S (2008) Incidence, aetiology and epidemiology of uterine fibroids. Best Pract Res Clin Obstet Gynaecol. 22 (4):571-588.

5. Schwartz SM, Marshall LM, Baird DD (2000) Epidemiologic contributions to understanding the etiology of uterine leiomyomata. Environ Health Perspect. 108 Suppl 5:821-827.

6. Wilde S, Scott-Barrett S (2009) Radiological appearances of uterine fibroids. Indian J Radiol Imaging. 19 (3):222-231.

7. Parker WH (2007) Etiology, symptomatology, and diagnosis of uterine myomas. Fertil Steril. 87 (4):725-736.

8. Downes E, Sikirica V, Gilabert-Estelles J et al (2010) The burden of uterine fibroids in five European countries. Eur J Obstet Gynecol Reprod Biol. 152 (1):96-102.
9. Borah BJ, Nicholson WK, Bradley L Stewart EA (2013) The impact of uterine leiomyomas: a national survey of affected women. Am J Obstet Gynecol. 209 (4):319.e1-20.

10. Havryliuk Y, Setton R, Carlow JJ et al (2017) Symptomatic fibroid management: systematic review of the literature. JSLS. 21 (3):e2017.00041. doi: https://doi.org/10.4293/JSLS.2017.00041.

11. Kim YS, Bae DS, Park MJ et al (2014) Techniques to expand patient selection for MRI-guided high-intensity focused ultrasound ablation of uterine fibroids. Am J Roentgenol 202 (2):443-451.

12. Jeong JH, Hong GP, Kim YR, Ha JE, Lee KS (2016) Clinical consideration of treatment to ablate uterine fibroids with magnetic resonance imaging-guided high intensity focused ultrasound (MRgFUS): sonalleve. J Menopausal Med. 22 (2):94-107.

13. Yu SC, Cheung EC, Leung VY, Fung LW (2019) Oxytocinaugmented and non-sedating high-intensity-focused ultrasound (HIFU) for uterine fibroids showed promising outcome as compared to HIFU alone or uterine artery embolization. Ultrasound Med Biol. 45 (12):3207-3213.

14. Sieroń D, Wiggermann P, Skupiński J Kukawska-Sysio K Lisek U, Koczy A (2011) Uterine artery embolisation and magnetic resonance-guided focused ultrasound treatment of uterine fibroids. Pol J Radiology. 76 (2):37-39.

15. Leonhardt H, Aziz A, Lönn L (2005) Post-embolization syndrome and complete expulsion of a leiomyoma after uterine artery embolization. Acta Obstet Gynecol Scand. 84 (3):303-305.

16. Rabkin BA, Zderic V, Crum LA Vaezy S (2006) Biological and physical mechanisms of HIFU-induced hyperecho in ultrasound images. Ultrasound Med Biol. 32 (11):1721-1729.

17. Haar GT, Coussios C (2007) High intensity focused ultrasound: physical principles and devices. Int J Hyperthermia. 23 (2):89-104.

18. Cunningham RK, Horrow MM, Smith RJ Springer J (2018) Adenomyosis: a sonographic diagnosis. Radiographics. 38 (5):1576-1589.

19. Fennessy FM, Tempany CM, McDannold NJ et al (2007) Uterine leiomyomas: MR imaging-guided focused ultrasound surgery-results of different treatment protocols. Radiology. 243 (3):885-893

20. Tempany CM, Stewart EA, McDannold N, Quade BJ, Jolesz FA, Hynynen K (2003) MR imaging-guided focused ultrasound surgery of uterine leiomyomas: a feasibility study. Radiology. 226 (3):897-905.

21. Funaki K, Sawada K, Maeda F, Nagai S (2007) Subjective effect of magnetic resonance-guided focused ultrasound surgery for uterine fibroids. J Obstet Gynaecol Res. 33 (6):834-839.

22. Hindman JC (1966) Proton resonance shift of water in the gas and liquid states. J Chem Phys. 44 (12):4582-4592.

23. Peters RD, Hinks RS, Henkelman RM (1998) Ex vivo tissue-type independence in proton-resonance frequency shift MR thermometry. Magn Reson Med. 40 (3):454-459.

24. Funaki K, Fukunishi H, Funaki T, Sawada K, Kaji Y, Maruo T (2007) Magnetic resonance-guided focused ultrasound surgery for uterine fibroids: relationship between the therapeutic effects and signal intensity of preexisting T2-weighted magnetic resonance images. Am J Obstet Gynecol. 196 (2):184. e1-6.

25. Brazier JE, Harper R, Jones NM et al (1992) Validating the SF-36 health survey questionnaire: new outcome measure for primary care. BMJ. 305 (6846):160-164.

26. Lee JS, Hong GY, Park BJ, Kim TE (2015) Ultrasound-guided high-intensity focused ultrasound treatment for uterine fibroid \& adenomyosis: a single center experience from the Republic of Korea. Ultrason Sonochem. 27:682-687.

27. LeBlang SD, Hoctor K, Steinberg FL (2010) Leiomyoma shrinkage after MRI-guided focused ultrasound treatment: report of 80 patients. Am J Roentgenol. 194 (1):274-280. 
28. Cheung VYT (2018) High-intensity focused ultrasound therapy. Best Pract Res Clin Obstet Gynaecol. 46:74-83.

29. Keserci B, Duc NM (2018) Magnetic resonance imaging parameters in predicting the treatment outcome of high-intensity focused ultrasound ablation of uterine fibroids with an immediate nonperfused volume ratio of at least 90. Acad Radiol. 25 (10):1257-1269.

30. Suomi V, Komar G, Sainio T, Joronen K, Perheentupa A, Blanco Sequeiros R (2019) Comprehensive feature selection for classifying the treatment outcome of high-intensity ultrasound therapy in uterine fibroids. Sci Rep. 9 (1):10907. doi: https://doi.org/10. 1038/s41598-019-47484-y.

31. Rein MS, Barbieri RL, Friedman AJ (1995) Progesterone: a critical role in the pathogenesis of uterine myomas. Am J Obstet Gynecol. 172 (1 Pt 1):14-18.

32. Chin W, Walbridge JR, Quinn SD (2015) MR-guided focused ultrasound for the treatment of adenomyosis: current insights. Res Rep Focused Ultrasound. 2016 (4):1-8. https://doi.org/10.2147/ RRFU.S95901.

33. Zhang L, Rao F, Setzen R (2017) High intensity focused ultrasound for the treatment of adenomyosis: selection criteria, efficacy, safety and fertility. Acta Obstet Gynecol Scand. 96 (6):707-714.

34. Jeng CJ, Ou KY, Long CY, Chuang L, Ker CR (2020) 500 cases of high-intensity focused ultrasound (HIFU) ablated uterine fibroids and adenomyosis. Taiwan J Obstet Gynecol. 59 (6):865-871.
35. Stewart EA, Rabinovici J, Tempany CM et al (2006) Clinical outcomes of focused ultrasound surgery for the treatment of uterine fibroids. Fertil Steril. 85 (1):22-29.

36. Mindjuk I, Trumm CG, Herzog P, Stahl R, Matzko M (2015) MRI predictors of clinical success in MR-guided focused ultrasound (MRgFUS) treatments of uterine fibroids: results from a single centre. Eur Radiol. 25 (5):1317-1328.

37. Moroni RM, Martins WP, Messina MDL et al (2016) Highintensity focused ultrasound for symptomatic uterine fibroids. Cochrane Database Syst Rev. https://doi.org/10.1002/14651858. CD012444.

38. Duc NM, Keserci B (2018) Review of influential clinical factors in reducing the risk of unsuccessful MRI-guided HIFU treatment outcome of uterine fibroids. Diagn Interv Radiol. 24 (5):283-291.

Publisher's Note Springer Nature remains neutral with regard to jurisdictional claims in published maps and institutional affiliations. 